\title{
BMJ open A botulinum toxin A treatment algorithm for de novo management of torticollis and laterocollis
}

\author{
Harald Hefter, ${ }^{1}$ Andreas Kupsch, ${ }^{2}$ Martina Müngersdorf, ${ }^{3}$ Sebastian Paus, ${ }^{4}$ \\ Andrea Stenner, ${ }^{5}$ Wolfgang Jost, ${ }^{6}$ on behalf of the Dysport Cervical Dystonia Study \\ Group*
}

To cite: Hefter $\mathrm{H}$, Kupsch $\mathrm{A}$, Müngersdorf $\mathrm{M}$, et al; on behalf of the Dysport Cervical Dystonia Study Group. A botulinum toxin A treatment algorithm for de novo management of torticollis and laterocollis. BMJ Open 2011:1:e000196. doi:10.1136/bmjopen2011-000196

- Prepublication history for this paper is available online. To view these files please visit the journal online (http:// bmjopen.bmj.com).

Received 27 May 2011 Accepted 21 June 2011

*The members of the Dysport Cervical Dystonia Study Group are listed in appendix 1.

This final article is available for use under the terms of the Creative Commons Attribution Non-Commercial 2.0 Licence; see http://bmjopen.bmj.com

For numbered affiliations see end of article.

Correspondence to Harald Hefter; harald.hefter@online.de

\section{ABSTRACT}

Objectives: Few studies have investigated the injection patterns for botulinum toxin type $A$ for the treatment of heterogeneous forms of cervical dystonia (CD). This large, prospective, open-label, multicentre study aimed to evaluate the effectiveness and safety of $500 \mathrm{U}$ botulinum toxin $\mathrm{A}$ for the initial treatment according to a standardised algorithm of the two most frequent forms of $C D$, predominantly torticollis and laterocollis.

Design: Patients (aged $\geq 18$ years) with CD not previously treated with botulinum neurotoxin therapy were given one treatment with $500 \mathrm{U}$ Dysport, according to a defined intramuscular injection algorithm based on clinical assessment of direction of head deviation, occurrence of shoulder elevation, occurrence of tremor (all evaluated using the Tsui rating scale) and hypertrophy of the sternocleidomastoid muscle.

Results: In this study, 516 patients were enrolled, the majority of whom $(95.0 \%)$ completed treatment. Most patients had torticollis $(78.1 \%)$. At week 4, mean Tsui scores had significantly decreased by $-4.01,-3.76$ and -4.09 points in the total, torticollis and laterocollis populations, respectively. Symptom improvement was equally effective between groups. Tsui scores remained significantly below baseline at week 12 in both groups. Treatment was well tolerated; the most frequent adverse events were muscular weakness $(13.8 \%)$, dysphagia (9.9\%) and neck pain (6.6\%). Conclusions: Dysport $500 \mathrm{U}$ is effective and well tolerated for the de novo management of a range of heterogeneous forms of $C D$, when using a standardised regimen that allows tailored dosing based on individual symptom assessment.

Clinical trials information: (NCT00447772; clinicaltrials.gov)

\section{INTRODUCTION}

Idiopathic cervical dystonia (CD) is the most frequent form of focal dystonia and is characterised by sustained involuntary muscle contraction and/or twitching of cervical musculature, resulting in head and neck

\section{ARTICLE SUMMARY}

Article focus

- Evaluation of the benefits of a treatment algorithm for the use of Dysport for the de novo management of torticollis and laterocollis in a large population of patients with cervical dystonia (CD)

Key messages

- Dysport $500 \mathrm{U}$ is effective and well tolerated for the de novo treatment of the majority of patients suffering from the most common forms of CD.

- The treatment algorithm proposed represents a clinically useful treatment algorithm to individualise Dysport treatment in approximately $90 \%$ of all CD subgroups.

Strengths and limitations of this study

- Strengths of this study include the larger patient population treatment.

- The injection protocol proposed can be useful to guide initial treatment in de novo patients with CD but should not replace clinical judgement and individual patient assessment.

movements as well as various undesired head positions. ${ }^{1}$ Depending on the direction of head movement, $\mathrm{CD}$ can be commonly classified as torticollis (turning of the head), laterocollis (head and neck tilt), retrocollis (head and neck extension), anterocollis (head and neck flexion) or a combination of the above. A more detailed analysis of the muscles that are involved in CD has recently been performed, taking into account whether the head or the neck is predominantly forced into abnormal positions, ${ }^{2}$ and this analysis may lead to new classification and terminology. However, this has yet to be widely adopted, and the former classification is still routinely used in clinical practice.

Botulinum neurotoxin (BoNT) has a longstanding and widespread use for the treatment of $\mathrm{CD}$ and is recommended as the first-line treatment option. ${ }^{3}$ The efficacy of 
botulinum toxin A (Dysport) for the treatment of CD has been demonstrated in multiple randomised, placebo-controlled trials. ${ }^{4-7}$ These studies have shown that Dysport $500 \mathrm{U}$ is an effective and well-tolerated starting dose for the treatment of $\mathrm{CD}$ and provides symptom relief for up to 3 months. ${ }^{4-7}$ Apart from one study specifically investigating the effects of Dysport on torticollis, ${ }^{4}$ the other studies have included a heterogeneous CD patient population with the number of injection sites and Dysport dose at each site determined based on investigator judgement. ${ }^{5-7}$ Careful selection of the dose of toxin used per muscle is essential, as inappropriate dosing can increase the risk of adverse events such as focal muscle weakness. ${ }^{8}$ Therefore, an injection protocol was predefined in this study, specifying dose and injection sites to minimise the risk of side-effects and systematically evaluate the effectiveness and safety of this dosing algorithm tailored to the individual heterogeneous subtypes of $\mathrm{CD}$. As such, this large, prospective, open-label, multicentre study aimed not only to confirm the effectiveness and safety of Dysport $500 \mathrm{U}$ for the initial treatment of the two most frequent forms of CD (predominantly torticollis and laterocollis) but also to evaluate the treatment algorithm used in this study (figure 1). This algorithm may help inexperienced users to inject Dysport effectively and safely.

\section{METHODS}

Study design and patients

This was a prospective, multicentre, open-label study conducted in Germany and Austria to investigate the effectiveness and safety of $500 \mathrm{U}$ Dysport for the treatment of heterogeneous forms of $\mathrm{CD}$. As botulinum toxin type A (BoNT-A) is regarded as a first-line neurological therapy for the treatment of $\mathrm{CD}$, this study did not include a placebo-control arm. The study was conducted in accordance with the Declaration of Helsinki, taking into account local regulatory requirements; all patients provided written informed consent to participate.

Male and female outpatients, aged $\geq 18$ years, with CD not previously treated with BoNT therapy, were eligible for inclusion. Exclusion criteria comprised patients with pure anterocollis or retrocollis, pure tremor capitis, or pure sagittal or lateral shifts, as these are rare complex forms of $\mathrm{CD}(<10 \%)$ and may require different treatment algorithms. Additional exclusion criteria included previous treatment with botulinum toxin for any indication other than $\mathrm{CD}$ within the past 12 months, as well as known antibodies to BoNT.

\section{Study treatment and treatment algorithm}

All patients received 500 U Dysport (Ipsen Biopharm, Wrexham, UK), diluted in $2.5 \mathrm{ml} 0.9 \% \mathrm{NaCl}$. Treatment was administered by intramuscular injections according to three predefined main decision steps. The investigator had to follow these steps and select (out of the 12 given injection protocols) the best suitable for the individual patient (figure 1):

1. main type of $\mathrm{CD}$ (either torticollis or laterocollis based on the Tsui score);

2. shoulder elevation ( $\geq 2$ Tsui score, subscore $\mathrm{C}$ ) or tremor (tremor, myoclonia, corresponding to Tsui score 4, subscore $\mathrm{D}$ ), which had to be treated as a second component;

3. presence of hypertrophy of the sternocleidomastoid muscle (marked vs light or no hypertrophy).

This decision cascade resulted in a corresponding injection protocol defining the dose and number of injection points per muscle (figure 1). The injection protocol for shoulder elevation was used when the patient had a shoulder elevation $\geq 2$ in Tsui subscore C. The injection protocol for tremor was used when the
Figure 1 Cervical dystonia types/subtypes and injection protocol. One vial of Dysport (500 U) is dissolved in $2.5 \mathrm{ml} 0.9 \%$ $\mathrm{NaCl}$ solution, the values given are the applied units. Plus shoulder elevation: $=2$ Tsui score, subscore C; Plus tremor: Tsui score 4, subscore D; Sternocleidomastoid muscle hypertrophy: yes (marked) or none/mild (light/no) hypertrophy. $\mathrm{CL}$, contralateral to movement direction; IL, ipsilateral to movement direction; SCM, sternocleidomastoid muscle.

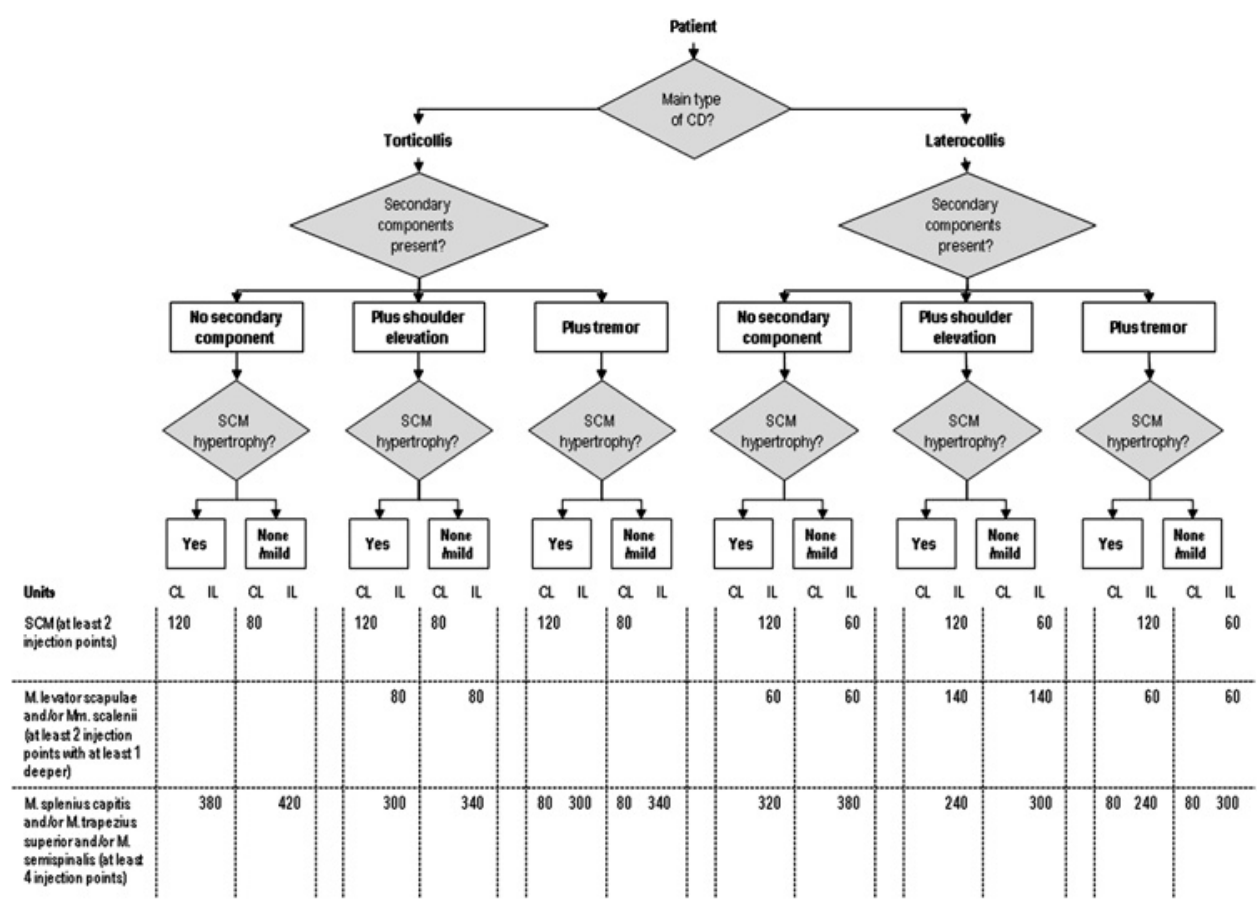


patients had a tremor score of 4 in Tsui subscore D. If the patient fulfilled both these criteria, it was the investigator's decision to treat the symptoms which were the most disabling for the patient and to use the corresponding injection protocol. Electromyography (EMG) guidance for injection was left to the discretion of the investigator.

\section{Assessments}

The decision rules follow a careful assessment of severity of CD symptoms using the Tsui scale ${ }^{9}$ under standardised conditions with the patient in a relaxed seated position. Assessment of $\mathrm{CD}$ symptom severity using the Tsui total score was repeated at weeks 4 and 12 post-treatment. The primary efficacy outcome was a change from baseline to week 4 in Tsui total score after treatment.

In addition, both investigators and patients provided a global assessment efficacy at weeks 4 and 12 posttreatment. This was rated on a four-point scale $(1=$ very good; $2=$ good; $3=$ moderate; $4=$ insufficient).

Safety assessments included incidence of AEs, neurological and physical examinations, vital signs and patient and investigator global assessment of tolerability, rated on a four-point scale (1=very good; 2=good; $3=$ moderate; $4=$ insufficient).

\section{Statistical analyses}

An original sample size of 600 subjects, enrolled over 24 months, was planned in order to detect a one-point between-treatment group difference on change from baseline to week 4 in Tsui total score with $90 \%$ power. However, this target was not reached, and as such, recruitment was stopped at 516 patients after 39 months. Following a data review, the primary statistical analysis plan was regarded as exploratory, and thus no adjustments for multiplicity were made. The safety population included all patients who received study medication and had at least one safety assessment. Effectiveness analyses were conducted on the intention-to-treat population, which included all patients in the safety sample who had at least one baseline and one post-treatment Tsui total score assessment. Additionally, confirmatory analyses using the per-protocol population (excluding major protocol violations) were conducted.

The primary effectiveness endpoint, the mean change in Tsui total score between baseline and week 4, was assessed by analysis of covariance (ANCOVA), with baseline Tsui total score as covariate and the main type of $\mathrm{CD}$ as between-group factor. Analyses were conducted for the total population and by main type of CD. The time course of the Tsui total score improvement was investigated by means of repeated-measures ANCOVA models, which included the main type of $\mathrm{CD}$ and week of assessment as the main effects and type of $\mathrm{CD}$ and week of assessment as covariates. The mean percentage improvement in Tsui total scores at weeks 4 and 12 was also evaluated for each main type of $\mathrm{CD}$; this analysis was specified post-hoc in order to facilitate comparison of the results with other studies. Safety data were analysed descriptively.

\section{RESULTS}

Patient disposition and demographics

A total of 516 patients were enrolled in this study at 81 study sites in Germany and Austria. The safety sample consisted of 515 patients; one patient received treatment but was excluded from the safety analysis, as no safety data were available. Patient disposition is shown in figure 2. Four hundred and eighty-nine patients (95.0\%) included in the safety sample completed the study.

Patient demographics and baseline characteristics are shown in table 1 . The majority of patients had torticollis $(\mathrm{n}=402 ; 78.1 \%) ; 112$ patients $(21.7 \%)$ had laterocollis; the type of $\mathrm{CD}$ was unknown in one patient. Baseline characteristics were similar between patients with torticollis and laterocollis, although the proportion of males was higher in patients with torticollis $(32.6 \%)$ compared with patients with laterocollis $(27.7 \%)$. More than half of patients $(56.5 \%)$ experienced pain associated with $\mathrm{CD}$, and $12.6 \%$ of patients had a documented additional sagittal or lateral shift. Twelve patients $(2.3 \%)$ reported dysphagia before treatment due to head deviation (torticollis $\mathrm{n}=7$ and laterocollis $\mathrm{n}=5$ ).

\section{Treatments and dosing}

All treated patients received Dysport $500 \mathrm{U}$ at baseline with the exception of three patients who received less than $500 \mathrm{U}$ (non-compliance to the injection protocol). All injections were given without EMG guidance. The most frequently reported concomitant medications ( $>10 \%$ of patients) by therapeutic class were betablocking agents (19.0\%), agents acting on the renin-angiotensin system (14.4\%), psychoanaleptics $(13.6 \%)$, thyroid therapy $(13.0 \%)$ and analgesics $(10.1 \%)$. Concomitant medication use was similar between $\mathrm{CD}$ types.

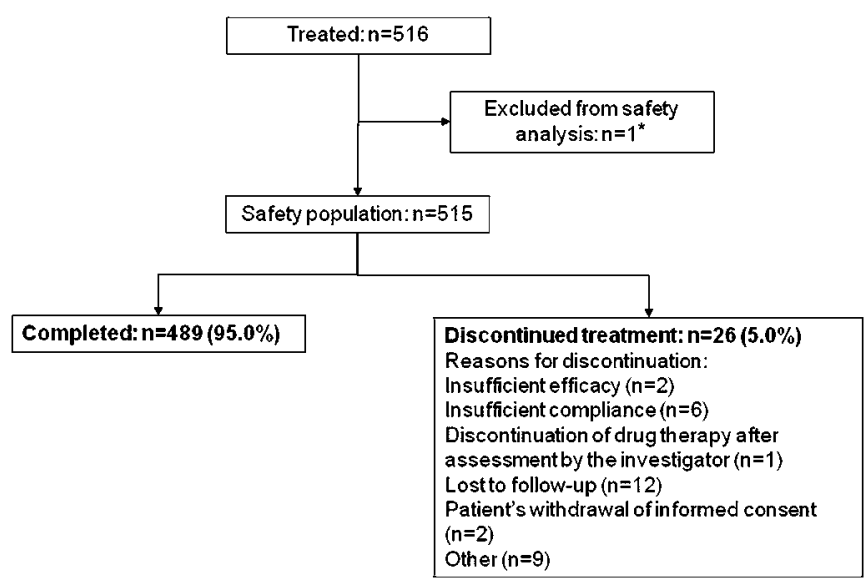

Figure 2 Patient disposition. *One patient was excluded from the safety analysis as treatment was discontinued prematurely due to insufficient compliance. No safety data was collected for this patient. 
Table 1 Baseline patient demographics: safety sample

\begin{tabular}{|c|c|c|c|}
\hline Parameter & Torticollis $(n=402)$ & Laterocollis $(n=112)$ & Total $^{*}(n=515)$ \\
\hline Gender, n (\%) male & $131(32.6)$ & $31(27.7)$ & $162(31.5)$ \\
\hline \multicolumn{4}{|l|}{ Age, years } \\
\hline Mean (SD) & $51.9(12.7)$ & $51.9(12.8)$ & $51.9(12.7)$ \\
\hline Range & $19-83$ & $19-87$ & $19-87$ \\
\hline Height, cm (mean (SD)) $\dagger$ & $169.1(8.6)$ & $168.4(8.7)$ & $169.0(8.6)$ \\
\hline Weight, kg (mean (SD))† & $73.2(15.4)$ & $70.7(12.1)$ & $72.6(14.7)$ \\
\hline $\mathrm{BMI}, \mathrm{kg} / \mathrm{m}^{2}$ (mean (SD)) $\dagger$ & $25.5(4.6)$ & $24.9(3.6)$ & $25.3(4.4)$ \\
\hline \multicolumn{4}{|l|}{ Race, n (\%) } \\
\hline Caucasian & $398(99.0)$ & $112(100.0)$ & $511(99.2)$ \\
\hline Asian & $1(0.2)$ & $0(0.0)$ & $1(0.2)$ \\
\hline Oriental & $3(0.7)$ & $0(0.0)$ & $3(0.6)$ \\
\hline \multicolumn{4}{|l|}{ Additional CD symptoms, $\mathrm{n}(\%)$} \\
\hline Pain $(p=0.0676 \ddagger)$ & $219(54.5)$ & $72(64.3)$ & $291(56.5)$ \\
\hline Shift (sagittal or lateral, $\mathrm{p}=0.0011 \ddagger$ ) & $40(10.0)$ & $25(22.3)$ & $65(12.6)$ \\
\hline Dysphagia $(p=0.1467 \ddagger)$ & $7(1.7)$ & $5(4.5)$ & $12(2.3)$ \\
\hline Other & $12(3.0)$ & $1(0.9)$ & $13(2.5)$ \\
\hline \multicolumn{4}{|l|}{ Subtypes of CD, $\mathrm{n}(\%) \S$} \\
\hline Without tremor/shoulder elevation $(p=0.0006 \ddagger)$ & $131(32.6)$ & $18(16.1)$ & $149(29.0)$ \\
\hline With shoulder elevation $(p=0.0024 \ddagger)$ & $126(31.3)$ & $53(47.3)$ & $179(34.8)$ \\
\hline With tremor $(p=0.9120 \neq)$ & $145(36.1)$ & $41(36.6)$ & $186(36.2)$ \\
\hline $\begin{array}{l}\text { Baseline total Tsui score (patient in sitting position), } \\
\text { mean (SD)§ }\end{array}$ & $8.4(3.5)$ & $8.2(3.3)$ & $8.4(3.5)$ \\
\hline
\end{tabular}

\section{Efficacy}

For the primary efficacy endpoint, Dysport significantly decreased mean Tsui total scores from baseline to week 4 $(-3.83 ; 95 \%$ CIs -4.01 to $-3.57 ; \mathrm{p}<0.0001)$ in the total population. Dysport also significantly decreased Tsui total scores from baseline to week 4 for patients with torticollis $(-3.76 ; 95 \%$ CI -4.02 to $-3.51 ; \mathrm{p}<0.0001)$ and patients with laterocollis $(-4.09 ; 95 \%$ CI -4.58 to $-3.59 ; \mathrm{p}<0.0001)$, corresponding to a percentage improvement of $43.7 \pm 36.4 \%$ and $46.5 \pm 28.3 \%$, respectively (total population: $44.3 \pm 34.8 \%$ ). The mean treatment difference between the torticollis and laterocollis groups was not statistically significant $(-0.32 ; 95 \%$ CI -0.88 to $0.23 ; p=0.255)$, indicating that both forms of CD equally improved at week 4 .

Significant improvements in Tsui total scores were sustained to week 12 for both CD types (figure 3), corresponding to similar percentage improvements in patients with torticollis $(23.6 \pm 44.6 \%)$ and laterocollis $(27.0 \pm 33.0 \%$ ) (total population $24.3 \pm 42.4 \%$ ).

Analysis of data using the per-protocol population $(n=490)$ confirmed findings from the intention-to-treat analysis $(n=503)$.

In analyses of the total population, the mean changes in Tsui subscale scores from baseline to week 4 were statistically significant for all Tsui subscores: amplitude of rotation, deflection (tilt) and ante-/retrocollis, subscore A: $-1.4 ; 95 \%$ CI -1.5 to -1.3 ; duration of movement, subscore B: -0.3 ; $95 \%$ CI -0.4 to -0.3 ; severity and duration of shoulder elevation, subscore C: $-0.4 ; 95 \%$
CI -0.5 to -0.3 ; and tremor, subscore D: $-0.6 ; 95 \% \mathrm{CI}$ -0.7 to -0.5$)$. The percentage improvement between the mean Tsui score at baseline and V2 was greatest for severity of tremor (45\%, subscore D) and least for duration of movement (22\%, subscore B). The percentage improvement in mean values between baseline and V2 was $40 \%$ each for subscores A (amplitude) and $\mathrm{C}$ (shoulder elevation), respectively.

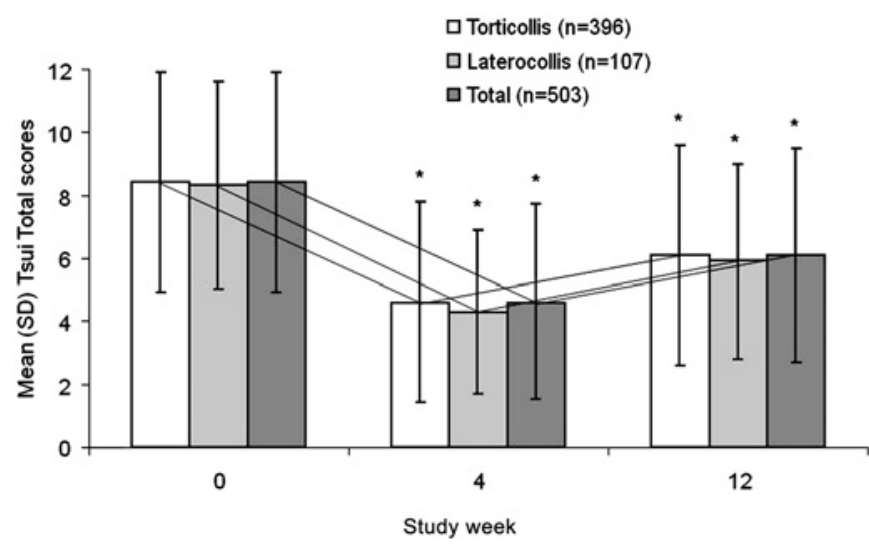

Figure 3 Mean Tsui total scores at baseline, week 4 and week 12 in the main CD subtypes and the total population (ITT population). ${ }^{*} p<0.0001$ vs baseline. The reduction of the Tsui total score was highly significant both at week 4 and 12 $(p<0.0001)$ and, as is evident from the parallel lines, nearly identical in the torticollis and laterocollis patient groups. CD, cervical dystonia; ITT, intention-to-treat. 
The efficacy of study medication in the total population was rated as 'very good' or 'good' by $70.0 \%$ of investigators (67.2\% torticollis, $80.2 \%$ laterocollis) and $60.8 \%$ of patients $(59.9 \%$ torticollis, $64.1 \%$ laterocollis) at week 4 . At week 12, efficacy was rated as 'very good' or 'good' by $72.0 \%$ of investigators $(70.4 \%$ torticollis, $77.9 \%$ laterocollis) and $64.9 \%$ of patients $(63.8 \%$ torticollis, $69.3 \%$ laterocollis).

\section{Safety and tolerability}

At least one $\mathrm{AE}$ was experienced by $41.4 \%$ of patients, of which $30.1 \%$ were considered to be related to study medication. The most frequent AEs ( $>5 \%$ of patients) were muscular weakness $(13.8 \%)$, dysphagia $(9.9 \%)$ and neck pain $(6.6 \%)$; no significant difference in the rates of these AEs was seen between patients with torticollis and laterocollis, except for severe muscular weakness (table 2). Most AEs were mild to moderate in severity $(89.7 \%)$; only 53 patients (10.3\%) experienced severe AEs. A summary of safety and tolerability by main type of $\mathrm{CD}$ is shown in table 2. Overall, AEs of muscular weakness, dysphagia and neck pain were rated as severe in 17 $(3.3 \%)$, three $(0.6 \%)$ and $13(2.5 \%)$ patients, respectively (table 2). One patient experienced severe dysphagia, severe muscular weakness and severe neck pain simultaneously, and five patients experienced both severe muscular weakness and severe neck pain. Nearly all cases of severe muscular weakness ( 15 of 17 patients; $88.2 \%$ ) and all cases of severe dysphagia (three patients) resolved without the requirement for intervention. Of the two patients with severe muscle weakness that were not classed as resolved, no information was available for one patient, and one patient had to wear a cervical collar temporarily. Of the patients with severe neck pain $(n=13)$, six $(46.2 \%)$ had pain that was self-limiting and did not require intervention, while the other seven $(53.8 \%)$ required intervention.

Eleven patients (2.1\%) experienced serious AEs (SAEs), although only two patients experienced serious AEs that were considered possibly related to study medication. One patient with torticollis experienced a convulsive syncope together with bradycardia immediately after injection; these symptoms resolved without intervention after several hours, and an EEG revealed no pathological findings. The attending physician assessed the events as probably being an injection-related, vagal reaction. One patient with laterocollis experienced muscle weakness of the head and depression: these symptoms developed 2 days post-treatment, and the patient was hospitalised 11 days post-treatment and treated with antidepressant medication. The patient recovered from both events within 3 weeks.

There were no relevant or unexpected observations in the physical and neurological examination or changes in vital signs with Dysport treatment. Tolerability of study medication, as assessed by investigators and patients, is summarised in table 2.

\section{DISCUSSION}

This study is the largest prospectively designed study conducted to date in de novo patients with $\mathrm{CD}$, as well as one of the largest studies conducted in patients with $\mathrm{CD}$ in general. The results of this study, conducted at multiple centres in Germany and Austria, demonstrate that a single dose of Dysport $500 \mathrm{U}$ can be used effectively for the management of the most common forms of

\begin{tabular}{|c|c|c|c|}
\hline & Torticollis $(n=402)$ & Laterocollis $(n=112)$ & Total $^{*}(n=515)$ \\
\hline \multicolumn{4}{|l|}{ Summary of AEs, $n(\%)$} \\
\hline Patients with AEs & $167(41.5)$ & $46(41.1)$ & $213(41.4)$ \\
\hline Patients with causally related $\mathrm{AE}$ & $121(30.1)$ & $34(30.4)$ & $155(30.1)$ \\
\hline Patients with at least one severe $A E$ & $35(8.7)$ & $18(16.1)$ & $53(10.3)$ \\
\hline Patients with SAE & $8(2.0)$ & $3(2.7)$ & $11(2.1)$ \\
\hline Patients with causally related SAE & $1(0.2)$ & $1(0.9)$ & $2(0.4)$ \\
\hline \multicolumn{4}{|c|}{ AEs in $>5 \%$ of patients in total population, $n(\%)$} \\
\hline Muscular weakness $(p=0.0618) \dagger$ & 49 (12.2) & $22(19.6)$ & $71(13.8)$ \\
\hline Severe $(p=0.0008) \dagger$ & $7(1.7)$ & $10(8.9)$ & $17(3.3)$ \\
\hline Dysphagia $(p=0.8582) \dagger$ & $41(10.2)$ & $10(8.9)$ & $51(9.9)$ \\
\hline Severe, $\mathrm{n}(\%)$ & $3(0.7)$ & $0(0.0)$ & $3(0.6)$ \\
\hline Neck pain $(p=0.8300) \dagger$ & $26(6.5)$ & $8(7.1)$ & $34(6.6)$ \\
\hline Severe, $\mathrm{n}(\%)$ & $9(2.2)$ & $4(3.6)$ & $13(2.5)$ \\
\hline \multicolumn{4}{|l|}{ Global assessment of tolerability } \\
\hline \multicolumn{4}{|c|}{ Percentage investigators rating tolerability as 'good' or 'very good' } \\
\hline Week 4 & 87.8 & 82.6 & 86.7 \\
\hline Week 12 & 89.2 & 87.9 & 88.8 \\
\hline \multicolumn{4}{|c|}{ Percentage patients rating tolerability as 'good' or 'very good' } \\
\hline Week 4 & 82.5 & 72.5 & 80.3 \\
\hline Week 12 & 85.7 & 84.1 & 85.4 \\
\hline
\end{tabular}

*Includes one patient in whom the main type of CD was unknown.

tFisher exact test for torticollis versus laterocollis, two-sided.

$\mathrm{AE}$, adverse event; $\mathrm{SAE}$, serious adverse event. 
CD (predominantly torticollis and laterocollis), when being injected according to a standardised algorithm that allows tailored dosing based on individual symptom assessment. Dysport treatment resulted in a clinically and statistically significant improvement in symptoms of $\mathrm{CD}$ at week 4 , as assessed by Tsui total scores, and the magnitude of improvement was comparable between torticollis and laterocollis patients. Furthermore, in both groups, the benefit of Dysport treatment was maintained to week 12.

The present results agree with previous studies that demonstrated the effectiveness of $500 \mathrm{U}$ Dysport for the treatment of $\mathrm{CD}^{4-7}$ and expand on these findings to demonstrate the comparable effectiveness of Dysport in the two main subtypes of CD. Dysport treatment in this study resulted in a greater than $40 \%$ improvement in CD symptoms at week 4 in all CD types, as measured by improvement in Tsui scores. This compares well with the percentage improvement in Tsui scores at week 4 in patients treated with Dysport in a double-blind placebo controlled study (Dysport $41 \%$ vs placebo $17 \%$; $\mathrm{p}=0.002)^{7}$

Improvements in symptoms were confirmed by investigator and patient global assessment of symptoms without distinct (significant) group differences. Of note, investigator and patient ratings varied most in the laterocollis group at week 4, which could probably be explained by the higher rate of severe muscular weakness reported in these patients.

The treatment protocol used in this study represents a clinically useful treatment algorithm to individualise treatment in approximately $90 \%$ of all CD subgroups, that is, torticollis and laterocollis, both with and without shoulder elevation or tremor, also accounting for hypertrophy of the sternocleidomastoid muscle. Independently, it is still essential for treating physicians to understand the biomechanical effects of the muscles involved in the primary movements of the head in order to optimise treatment when using this algorithm. The majority of patients with $\mathrm{CD}$ may be treated by the present investigated, standardised injection protocol. However, this protocol may not be suitable for patients with rare, more complex forms of CD. Thus, injection algorithms may facilitate effective dosing of BoNT-A, as excess dosing into muscles not involved in $\mathrm{CD}$ symptoms may not improve efficacy but rather increase the risk of AEs. The findings of this study support the use of one vial of $500 \mathrm{U}$ Dysport as an appropriate starting dose for patients with $\mathrm{CD}^{4-7}$ and comply with findings of a recent long-term follow-up of Dysport in $\mathrm{CD}$, suggesting that the majority of patients can benefit from this dose over the longer term, given a careful injection technique. ${ }^{10}$ The algorithm presented allows the optimal distribution of one $500 \mathrm{U}$ vial of Dysport based on the patient's clinical picture, assessed by the Tsui score, and is practicable in a normal clinical setting.

Dysport was well tolerated for the treatment of CD in this study; most AEs were mild to moderate in severity, and the majority of patients and investigators rated treatment tolerability as very good/good, regardless of torticollis or laterocollis. Interestingly, and as seen in the efficacy ratings, investigator and patient ratings of tolerability varied most in the laterocollis group at week 4 . It is possible that the statistically significant difference in severe muscular weakness between torticollis and laterocollis patients explains why laterocollis patients rated their tolerability of study treatment lower than torticollis patients at week 4 . No new safety concerns were raised by this study, and the most common AEs reported, muscular weakness and dysphagia, were consistent with the known safety profile of this medication in this indication. $^{11}$

The reported rates of muscle weakness and dysphagia were consistent with those reported for Botox in de novo patients, ${ }^{12}$ although there are inherent limitations in comparing data, assessment methods and botulinum toxin A formulations between studies. Rates of dysphagia and muscular weakness presented here are lower than rates reported in previously conducted double-blind, placebo-controlled trials with Dysport, ${ }^{56}$ probably due to dose distribution of Dysport based on a predefined injection protocol. Specifically, with regard to dysphagia, the majority of the BoNT-A dose in this study was injected into the posterior part of the neck region. Thus, the results show that the use of predefined injection protocols allowing individual symptom treatment may have the potential to improve treatment tolerability by providing patients with effective symptom relief while possibly limiting AEs associated with injecting noninvolved muscles. Finally, it is important to point out that the injection protocol may guide the initial treatment in de novo patients with $\mathrm{CD}$. However, the dose and muscle selection of further injections should always consider the patient's individual symptoms in conjunction with the initial treatment outcomes. Careful and extensive clinical examination and diagnosis are essential in all patients, especially those presenting with symptoms of pain and dysphagia caused by head deviation.

In conclusion, Dysport $500 \mathrm{U}$ is effective and well tolerated for the de novo treatment of the majority of patients suffering from the most common forms of CD. Analyses of additional secondary effectiveness outcomes collected in this study will provide further insight into the benefits of Dysport in this de novo patient group.

\section{Author affiliations}

${ }^{1}$ Department of Neurology, University of Düsseldorf, Moorenstrasse, Düsseldorf, Germany

${ }^{2}$ Department of Neurology, University Hospital Charité, Campus Virchow-Klinkum, Augustenburger Platz, Berlin, Germany

${ }^{3}$ Neurologie am Hackeschen Markt, Dircksenstr, Berlin, Germany

${ }^{4}$ Department of Neurology, University of Bonn, Sigmund-Freud-Strasse, Bonn, Germany

${ }^{5}$ Out-Patient Department of Neurology, Paracelsus Klinik, Werdauer Strasse, Zwickau, Germany

${ }^{6}$ Department of Neurology, Deutsche Klinik für Diagnostik Wiesbaden, Aukammallee, Wiesbaden, Germany 
Acknowledgements Data management and statistical analyses were performed by GKM Gesellschaft für Therapieforschung in Munich, Germany. Editorial assistance for the preparation of this manuscript was provided by Ogilvy Healthworld Medical Education. The authors would like to thank C Colling, Medical Project Manager, Ipsen Pharma GmbH, for her assistance in the conduct of the study and for reviewing the manuscript.

Correction notice The "To cite: ..." information and running footer in this article have been updated with the correct volume number (volume 1).

Funding Funding was provided by Ipsen Pharma, Germany.

Competing interests $\mathrm{HH}$ : consultant and speaker for Ipsen; AK: speaker honoria from Ipsen; MM: speaker for Ipsen; SP: consultant and speaker for Ipsen; AS: speaker for Ipsen; WJ: consultant and speaker for Ipsen.

Patient consent Obtained.

Ethics approval Ethics approval was provided by Ethics Committee of the Medical Faculty of the Heinrich-Heine University of Dsseldorf, Moorenstrasse, Dsseldorf, Germany (Lead EC) and local Ethics Committees.

Contributors $\mathrm{HH}$ and WJ were involved in the concept and study design, study conduct, data collection, analysis and interpretation, manuscript writing, review and critique. All other authors were involved in conducting the study, data collection, manuscript review and critique. All authors approved the final manuscript.

Provenance and peer review Not commissioned; externally peer reviewed.

Data sharing statement Data management and statistical analyses were performed by GKM Gesellschaft für Therapieforschung in Munich, Germany.

\section{REFERENCES}

1. Van Zandijcke M. Cervical dystonia (spasmodic torticollis). Some aspects of the natural history. Acta Neurol Belg 1995;95:210-15

2. Reichel G, Stenner A, Jahn A. Zur Phänomenologie der zervikalen Dystonien [The phenomenology of cervical dystonia]. Fortschr Neurol Psychiatr 2009;77:272-7.

3. Simpson DM, Blitzer A, Brashear A, et al. Botulinum neurotoxin for the treatment of movement disorders (an evidence-based review): report of the Therapeutics and Technology Assessment Subcommittee of the American Academy of Neurology. Neurology 2008;70:1699-706.

4. Poewe W, Deuschl G, Nebe A, et al. What is the optimal dose of botulinum toxin $A$ in the treatment of cervical dystonia? Results of a double blind, placebo controlled, dose ranging study using Dysport. German Dystonia Study Group. J Neurol Neurosurg Psychiatry 1998;64:13-17.

5. Truong D, Duane DD, Jankovic J, et al. Efficacy and safety of botulinum type A toxin (Dysport) in cervical dystonia: results of the first US randomized, double-blind, placebo-controlled study. Mov Disord 2005;20:783-91.

6. Truong $\mathrm{D}$, Brodsky M, Lew $\mathrm{M}$, et al. Long-term efficacy and safety of botulinum toxin type A (Dysport) in cervical dystonia. Parkinsonism Relat Disord 2010;16:316-23.

7. Wissel J, Kanovsky P, Ruzicka E, et al. Efficacy and safety of a standardised 500 unit dose of Dysport (Clostridium botulinum toxin type A haemaglutinin complex) in a heterogeneous cervical dystonia population: results of a prospective, multicentre, randomised, doubleblind, placebo-controlled, parallel group study. J Neurol 2001:248:1073-8.
8. Walker FO. Botulinum toxin therapy for cervical dystonia. Phys Med Rehabil Clin N Am 2003;14:749-66.

9. Tsui JK, Eisen A, Stoessl AJ, et al. Double-blind study of botulinum toxin in spasmodic torticollis. Lancet 1986;2:245-7.

10. Mohammadi B, Buhr N, Bigalke $\mathrm{H}$, et al. A long-term follow-up of botulinum toxin A in cervical dystonia. Neurol Res 2009;31:463-6.

11. Costa J, Espirito-Santo C, Borges A, et al. Botulinum toxin type A therapy for cervical dystonia. Cochrane Database Syst Rev 2005:CD003633.

12. Brin MF, Comella CL, Jankovic J, et al. Long-term treatment with botulinum toxin type $A$ in cervical dystonia has low immunogenicity by mouse protection assay. Mov Disord 2008;23:1353-60.

\section{APPENDIX 1}

\section{Dysport Cervical Dystonia Study Group}

Members of the Dysport Cervical Dystonia Study Group in Germany were (in alphabetical order of study sites): $\mathrm{H}$ Eing (Ahaus); A Plewe (Apolda); B Patzner (Arnsdorf); H Griese (Bad Oeyenhausen); M Pott (Bayreuth); A Nebe, T Winter and J Wissel (Beelitz-Heilstätten); H Woldag (Bennewitz); W Raffauf (Berlin); K Tiel-Wilck (Berlin); AM Garcia, D Gruber, F Kempf, H Krug, E Lobsien (Berlin); S Leistner (Berlin); A Schenkel-Römer (Berlin); S Eue (Bernburg); M Bonse (Bielefeld); I Nastos (Bochum); S Otto (Bochum); T Grehl and M Tegenthoff (Bochum); M Ebke (Bremen); E Kunesch, T Probst and M Richter (Deggendorf); A Hermann and M Wolz (Dresden); U Kahlen and M Moll (Düsseldorf); A Grüger (Eberswalde); P Feise, H W Kölmel and L Kotthaus (Erfurt); M Haslbeck (Erlangen); A Jaspert-Grehl (Essen); M Maschke, M Obermann and N Putzki (Essen); J Böhm (Freiberg); C Schiel (Gießen), M Hahn (Gießen), I Reuter and S Schmidt (Gießen); H Krumpolt (Großschweidnitz); FM Brake (Hagen); F Hoffmann and C Wohlrab (Halle-Dölau); V Becker and T Humbert (Hamburg); B Mohammadi (Hannover), R Dengler, K Kollewe, K Krampfl and S Petri (Hannover); J Osterhage (Homburg); D Müller (IImenau); K Gehring (Itzehoe); G Heide and A Jentsch (Jena); M Morgenthaler (Kaiserslautern); J Volkmann (Kiel); U Marczynski and J Wöhrle (Koblenz); B Wittmann (Landshut); S Peschel (Leipzig); P Baum (Leipzig); R Schneider (Lemgo); J Hagenah (Lübeck); K-A Bohr (Lüneburg); H Bäzner and C Blahak (Mannheim); J Philipps (Minden); R Althoff, J Haan and K Kessler (Mönchengladbach); F Castrop, C Dresel, L Esposito, C Loer and B Haslinger (München), AO Ceballos-Baumann, U Fietzek, M Messner and L Riedl (München); $M$ Ritter and $P$ Young (Münster); $F$ Erbguth (Nürnberg), R Witte (Oldenburg); M Putzer (Paderborn); H Kursawe (Potsdam); K Stutterheim (Potsdam); J Vollmer-Haase (Recklinghausen); R Benecke (Rostock), M Schöffel (Rüdersdorf); E Fleischer (Schorndorf); A Nguento (Schwedt/Oder); A Bartels (Schwerin); T Warnecke (Seesen); H Roick (Singen); M Petrick (Teupitz); J Hahn (Weiden); E Fucik, S Heitmann and B Zeiler (Wiesbaden); P Cordes and J Schierenbeck (Wolfenbüttel); C Helbig, M Klein and K-U Oehler (Würzburg); M Beck and A Schramm (Würzburg); G Hennen (Wuppertal); G Reichel (Zwickau).

Members of the Dysport Cervical Dystonia Study Group in Austria were (in alphabetical order of study sites): J Diez (Graz); P Schnider (Grimmenstein-Hochegg); T Haydn, J Müller and W Poewe (Innsbruck); RJ Resch and R Sommer (Linz). 